Check for updates

Cite this: Mater. Adv., 2021, 2, 6058

Received 15th June 2021, Accepted 10th August 2021

DOI: 10.1039/d1ma00516b

rsc.li/materials-advances

\section{Rational design of a NIR-II fluorescent nanosystem with maximized fluorescence performance and applications}

\begin{abstract}
Haoli Yu, Yuesong Wang, Yan Chen and Min Ji D *
Near-infrared (NIR) fluorescence imaging (FI) has become a research hotspot in the field of in vivo imaging. Here, we intend to synthesize a NIR-II fluorescent nanosystem with excellent fluorescence performance to achieve high-resolution and long circulating vascular imaging performance in vivo. In this work, fluorescence liposomes with differently charged phospholipids and various amounts of IR-1061 were designed and synthesized for analysing the encapsulation effect and conformational changes of IR-1061 within liposomes. Furthermore, an IR-1061 liposome (IR1061-ALP-N3) with optimal fluorescence intensity was prepared to study its in vivo $\mathrm{Fl}$ effect as well as the long circulation angiographic effect. The experimental results demonstrated that cationic liposomes showed the worst encapsulation effect on IR-1061 and anionic liposomes showed the best, which was consistent with the electrostatic interactions between phospholipids and IR-1061. Next, we found that IR-1061 possessed two states as a function of its concentration in liposomes: the free state and aggregate state. The aggregate state caused by the increase of IR-1061 content will decrease the fluorescence properties of the IR-1061 liposome. In vivo FI showed that IR1061-ALP-N3 exhibited excellent fluorescence performance in mice and successfully achieved clear systemic angiography with excellent spatial resolution. Moreover, long-term imaging also proved that IR1061-ALP-N3 can maintain high fluorescence intensity in vessels for more than 16 hours, indicating its angiographic function in the long circulation. The encapsulation effect of IR-1061 in liposomes was proved to be tightly related to the charge of phospholipids, and the limited space within liposomes would significantly affect the conformational changes of IR-1061. These results indicate that the rational design of IR-1061 liposomes with appropriate electrostatic effects and drug concentrations is an essential prerequisite to achieving high fluorescence performance. The excellent imaging effect and long circulation angiography contributed by this nanosystem also provide more favorable application prospects of NIR-II fluorophores for intravital imaging.
\end{abstract}

\section{Introduction}

Fluorescence imaging (FI) has been widely applied in various biological detection processes such as DNA sequencing, immunoassay, gene detection and cell imaging because of its simple operation, high sensitivity and the absence of radioactivity. ${ }^{1-3}$ Research studies have shown that the near-infrared II region (NIR-II: 1000-1700 nm) FI possesses lower photon scattering and autofluorescence background, making it promising for deep tissue imaging. ${ }^{4}$ These advantages make NIR-II FI an important technique widely applied in the field of in vivo

State Key Laboratory of Bioelectronics, Jiangsu Laboratory for Biomaterials and Devices, School of Biological Science and Medical Engineering, Southeast University, Nanjing, 210096, China. E-mail: 101010516@seu.edu.cn imaging such as intraoperative imaging, hemodynamic evaluation and precise quantification of abnormal femoral arteries. ${ }^{5,6}$

At present, inorganic NIR-II fluorescent materials such as quantum dots, single-walled carbon nanotubes, and rare-earthdoped nanoparticle materials have been extensively studied. ${ }^{7-9}$ Inorganic materials possess excellent optical properties, but their biocompatibility is still challenged by their slow excretion kinetics and long-term retention in the reticuloendothelial system. ${ }^{10}$ In contrast, the advantages of organic small molecular fluorophores in terms of biocompatibility and safety make them more suitable for clinical translation. ${ }^{11}$ For now, cyanine and D-A-D structures are the main types of NIR-II organic fluorophores, and each of them possesses relative merits. Cyanine has an extremely high molar extinction coefficient ( $\varepsilon)$ value, but its fluorescence QY and photostability are terrible because of the presence of its unstable multiple 

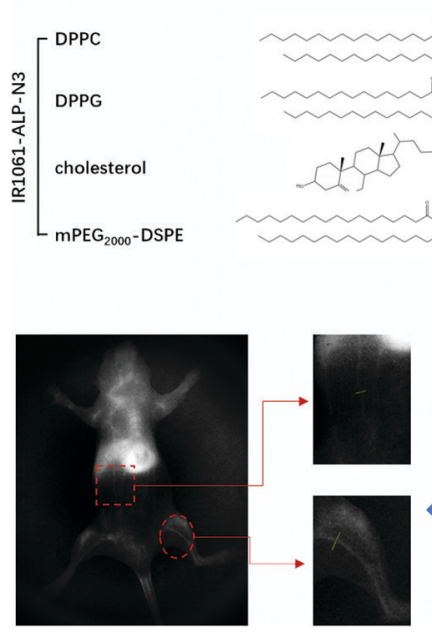

Fig. 1 Schematic overview of experimental procedures. The rational design of liposomes with high fluorescence properties and the imaging performance of angiography in vivo.

methyl chains. ${ }^{12}$ The D-A-D fluorophore, acclaimed for its stability and high QY, suffers from a poor $\varepsilon$ value. ${ }^{13}$

Moreover, both cyanine and D-A-D fluorophores contain a huge hydrophobic conjugated structure, resulting in poor solubility in vivo. ${ }^{14}$ Some strategies such as modifying hydrophilic groups on these fluorophores may also cause other problems like polar solvent effects or intramolecular charge transfer (ICT) destruction, which will weaken their fluorescence performance. ${ }^{15,16}$ In contrast, coating fluorophores with suitable carriers can, to a great extent, circumvent the complex interactions between fluorophores and solvents. ${ }^{17}$ IR-1061 is a kind of hydrophobic cyanine with a fluorescence peak at $1064 \mathrm{~nm}$, and its QY value $(1.70 \pm 5 \%)$ is higher than those of normal carbon nanotubes (about 0.4\%) and most other cyanine fluorophores, making it suitable for preparing NIR-II fluorescent nanosystems. ${ }^{18,19}$

In our previous research, we successfully encapsulated indocyanine green (ICG) and IR-1061 with lecithin to obtain enhanced NIR-II fluorescence signals in vivo. ${ }^{20}$ Unexpectedly, we found that the charge properties of liposomes were able to significantly affect their fluorescence properties. In this work, we investigated the effect of the charge properties of liposomes on the encapsulation effect of IR-1061 by preparing fluorescence liposomes with three different charges of synthetic phospholipids. The spatial arrangement caused by the change of the IR-1061 concentration in liposomes was also studied to realize the reasonable design of IR-1061 liposomes that can maximize their fluorescence intensity. Furthermore, the NIR-II fluorescence signals and the characteristics of long blood circulation of IR-1061 liposomes were studied to assess their imaging performance in vivo (Fig. 1).

\section{Experimental}

\subsection{Materials}

Cholesterol, methylated polyethylene glycol-stearoyl phosphatidylethanolamine ( $\left.\mathrm{mPEG}_{2000}-\mathrm{DSPE}\right)$, dipalmitoyl phosphatidylcholine
(DPPC), dipalmitoyl phosphatidylglycerol (DPPG) and 2,3-dioleoylpropyl-trimethylamine (DOTAP) were obtained from Jiangsu Southeast Nanomaterials co., Ltd (Jiangsu, China). IR-1061, chloral hydrate and 3-(4,5-dimethylthiazol-2-yl)-2,5-diphenyl tetrazolium bromide (MTT) were purchased from Sigma-Aldrich (Shanghai, China). Calcein-AM/PI Double Staining Kit, Annexin V-FITC/PI Apoptosis Detection Kit, trypsin and phosphate buffer saline (PBS) were purchased from KeyGen Biological Technology Co., Ltd (Nanjing, China). A549 cells were obtained from American Type Culture Collection (ATCC). Balb/c nude mice (female, 6-7 weeks) were purchased from the Nanjing Qinglongshan Animal Breeding Farm (Jiangsu, China).

\subsection{Preparation of NIR-II imaging liposomes}

Liposomes comprise IR-1061, cholesterol, $\mathrm{mPEG}_{2000}-\mathrm{DSPE}$ and various synthetic phospholipids. Neutral liposomes adopted the uncharged DPPC phospholipids singly, and cationic (anionic) liposomes were prepared with an equal molar ratio of DPPC and DOTAP (DPPG).

All liposomes were prepared by the method of thin film hydration sonication. ${ }^{21}$ Specifically, phospholipid: cholesterol: $\mathrm{mPEG}_{2000}-\mathrm{DSPE}(30: 10: 3, \mathrm{~m} / \mathrm{m})$ was prepared and dissolved in a dichloromethane/ethanol mixture $(2: 1, \mathrm{v} / \mathrm{v})$. The mixed solution was gradually removed by gradient decompression using a rotary evaporator under $60{ }^{\circ} \mathrm{C}$ to form a uniform film. Afterward, the film was fully hydrated in PBS to a phospholipid concentration of $10 \mathrm{mg} \mathrm{mL} \mathrm{m}^{-1}$. The original liposome solution was then sonicated with an ultrasound probe $(600 \mathrm{w}, 20 \%$ amplitude) and then filtered using a $0.22 \mu \mathrm{m}$ water syringe filter to form even IR-1061 liposomes.

\subsection{Physicochemical characterization of NIR-II imaging liposomes}

The mean hydrodynamic size and $\zeta$-potential of liposomes were measured using a Malvern Zetasizer Nano instrument (Malvern Instruments Ltd, Malvern, UK). The morphology 
and size of liposomes were obtained using a transmission electron microscope (TEM).

The content of IR-1061 in liposomes was calculated by the entrapment efficiency (EE) and the loading efficiency (LE) of liposomes was measured by spectrophotometry. Specifically, the absorption of IR-1061 at $1061 \mathrm{~nm}$ in methanol was detected using a UV3600 UV-Vis-NIR spectrophotometer (Shimadzu, Kyoto, Japan), and a linear simulation of the relationship between the concentration and absorbance was performed as a standard curve. The concentrations of IR-1061 in liposomes were then determined by the standard curve.

\subsection{In vitro NIR fluorescence spectrum analysis}

The absorption spectra of liposomes were recorded using a UV-Vis-NIR spectrophotometer under an appropriate concentration. The NIR-II fluorescence emission spectra were acquired using a low-temperature time-resolved fluorescence spectrometer FLS980 (Edinburgh Instruments, Edinburgh, UK). The emission spectra (1000-1200 nm) were collected under excitation at $980 \mathrm{~nm}$.

\subsection{In vitro NIR-II imaging}

The in vitro fluorescence intensity of fluorescent liposomes was detected using a small animal NIR-II biological imaging system (Wuhan Grand Imaging Technology Co., Ltd, China). Specifically, liposomes with different concentrations were placed into 96-well plates (200 mL per well) and excited with a $1064 \mathrm{~nm}$ laser. The emitted fluorescence was captured using a NIR-II camera with a $1064 \mathrm{~nm}$ long pass filter (1064 LP) and a $1064 \mathrm{~nm}$ cut-off filter (1064 OD) under an exposure time of $300 \mathrm{~ms}$. The 1064 OD filter can cut off the wavelength at $1064 \mathrm{~nm}$ and avoid the background interference of the excitation wavelength.

The tissue penetration depth of fluorescent liposomes was evaluated using chicken tissue in vitro. ${ }^{22}$ First, a small number of liposomes were injected into a capillary tube, and then covered with the chicken breast which was cut into small pieces with uniform size but varying thicknesses of $1 \mathrm{~mm}, 2 \mathrm{~mm}$, $4 \mathrm{~mm}, 6 \mathrm{~mm}$ and $8 \mathrm{~mm}$. The fluorescence intensity of liposomes was detected using a small animal NIR-II biological imaging system under the same conditions mentioned above.

\subsection{Cytotoxicity assay}

Cell viability was determined by MTT. A549 cells $\left(1 \times 10^{4}\right.$ cells per well) were plated onto 96-well plates with $100 \mu \mathrm{L}$ medium and cultured for 12 hours in a $\mathrm{CO}_{2}$ incubator $\left(37{ }^{\circ} \mathrm{C}, 5 \% \mathrm{CO}_{2}\right)$. After that, $100 \mu \mathrm{L}$ of IR-1061 liposome solution with different concentrations was added and kept for 12 hours and then $20 \mu \mathrm{L}$ of MTT $\left(5 \mathrm{mg} \mathrm{mL}^{-1}\right.$ ) per well was added and cultured for another 4 hours. The medium in 96-well plates was carefully replaced by $150 \mu \mathrm{L}$ of dimethyl sulfoxide (DMSO) and vibrated until the crystals dissolved. The absorbance of A549 cells was measured with a microplate reader (TECON infinite 200 Pro; Switzerland) at $490 \mathrm{~nm}$.

The living/dead cell count was evaluated with the CalceinAM/PI Double Staining Kit. A549 cells $\left(1 \times 10^{5}\right.$ cells per well $)$ were plated onto 6-well plates with $2 \mathrm{~mL}$ medium and cultured for 12 hours in a $\mathrm{CO}_{2}$ incubator $\left(37^{\circ} \mathrm{C}, 5 \% \mathrm{CO}_{2}\right)$. After that, 1 $\mathrm{mL}$ of IR-1061 liposomes with different concentrations were added and kept for 12 hours. A549 cells were then collected using trypsin and washed with PBS three times. Cell staining was performed in accordance with the instructions of the kit. The staining of living/dead cells was observed using a fluorescence inverted microscope (Olympus, Japan).

The A549 cell apoptosis was detected using the Annexin V-FITC/PI Apoptosis Detection Kit and all the experimental steps were consistent with the above ones. The staining of A549 cells was detected by flow cytometry (Thermo Scientific, USA).

\subsection{In vivo NIR-II fluorescence imaging}

All in vivo procedures were performed under a protocol approved by the Institutional Animal Care and Use Committee of Southeast University. Studies were conducted in Balb/c female mice (25 $\pm 2 \mathrm{~g}, 6-7$ weeks) from Qinglongshan Biosciences. Before imaging, mice were anesthetized by intraperitoneal injection of $7 \%$ chloral hydrate $\left(5 \mathrm{~mL} \mathrm{~kg}^{-1}\right)$ and then $0.2 \mathrm{~mL}$ of liposome solution $\left(10 \mathrm{mg} \mathrm{mL}^{-1}\right)$ was injected intravenously. The laser source power density at the imaging stage was $40 \mathrm{~mW} \mathrm{~cm} \mathrm{~cm}^{-2}$, excited with a $1064 \mathrm{~nm}$ laser. The emitted fluorescence was captured using a NIR-II camera with 1064 LP and 1064 OD filters under an exposure time of $3000 \mathrm{~ms}$.

\subsection{Biodistribution assay}

After the intravenous injection of liposomes for 24 hours, the mice were dissected and their heart, liver, spleen, lungs, kidneys and intestines were removed. The liposome content of each organ was qualitatively analyzed by fluorescence intensity detection (excitation: $1064 \mathrm{~nm}$, exposure time: $300 \mathrm{~ms}$ ).

\subsection{Data analysis}

Data are expressed as mean \pm standard deviation (SD). The in vitro NIR-II fluorescence intensities were measured with a small animal NIR-II biological imaging system, aligned and analyzed using GraphPad Prism software. Analyses of in vitro and in vivo NIR-II fluorescence images were performed using ImageJ software. The SBR was measured and quantified by grayscale analysis.

\section{Results}

\subsection{Physicochemical and photophysical characterization of IR-1061 liposomes}

To investigate the effect of phospholipid charge on the encapsulation of IR-1061, three types of liposomes with different phospholipid species were designed and synthesized. Neutral liposomes (NLPs) adopted the uncharged DPPC phospholipids, while cationic liposomes (CLPs) and anionic liposomes (ALPs) additionally added positively charged DOTAP and negatively charged DPPG phospholipids. As shown in Fig. 2a, the mean hydrodynamic size of the three liposomes was $85 \mathrm{~nm}$ 

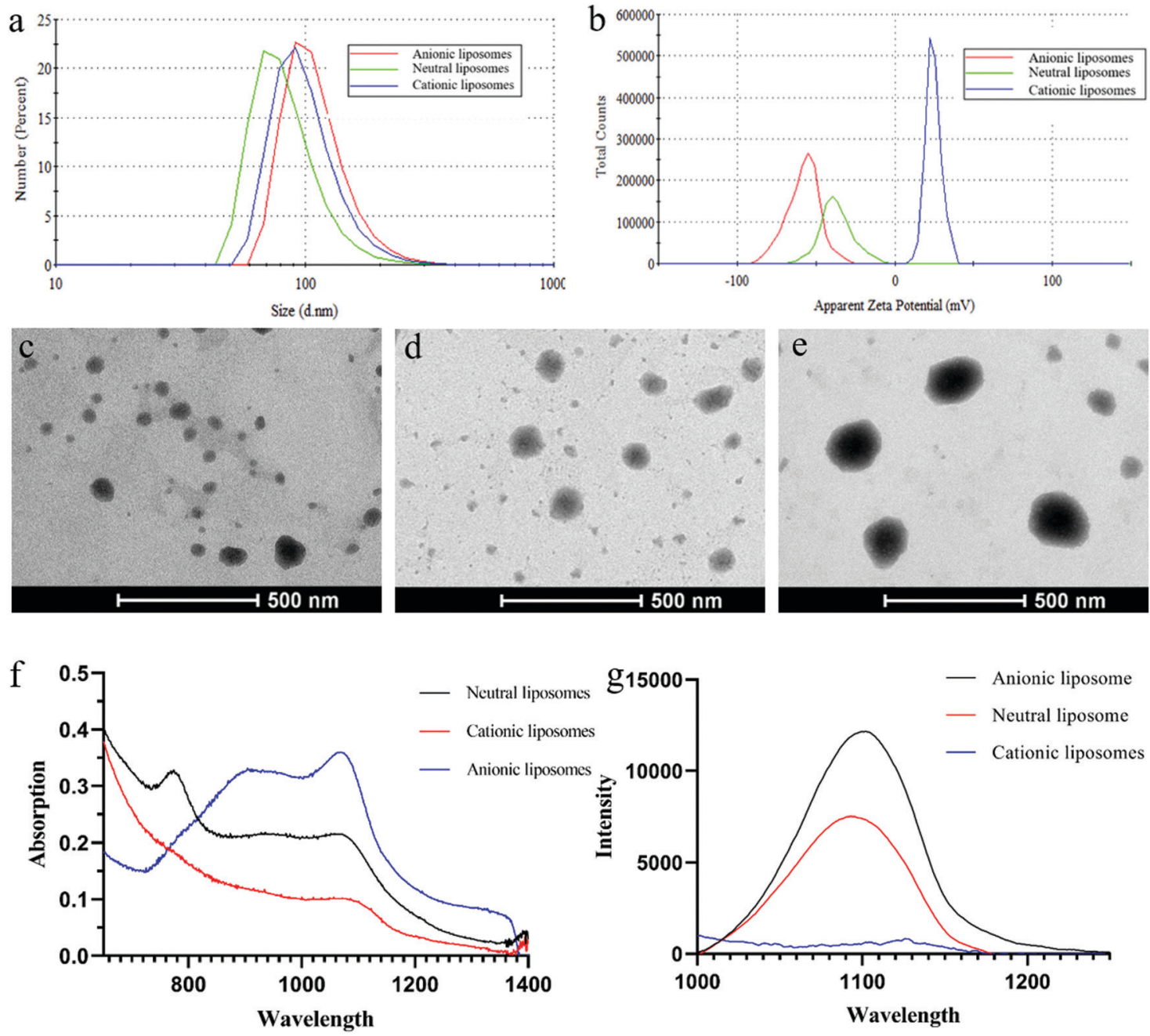

Fig. 2 Physicochemical and photophysical characterization of IR-1061 liposomes. The mean hydrodynamic sizes (a), $\zeta$ potential values (b) and TEMs (c-e) of three differently charged (neutral, cationic and anionic) liposomes. (f) The absorption of three differently charged liposomes. (g) The fluorescence intensity of three differently charged liposomes under $1061 \mathrm{~nm}$ excitation.

(PDI: 0.138), $102 \mathrm{~nm}$ (PDI: 0.208) and $112 \mathrm{~nm}$ (PDI: 0.125). The TEM results demonstrated that all liposomes displayed a roughly spherical morphology with a homogeneous distribution of particles (Fig. 2c-e). The $\zeta$ potential of CLPs confirmed that the liposome surface was indeed positively charged, and the absolute value of the $\zeta$ potential of all liposomes was greater than $20 \mathrm{mV}$ which indicated good colloidal stability (Fig. 2b).

The absorption and fluorescence intensities of all liposomes were compared at 1\% IR-1061 content (Fig. 2f and g). At $1061 \mathrm{~nm}$, the absorption and fluorescence intensities of ALPs were the highest and those of CLPs were the lowest. NLPs have a sharp absorption peak at $770 \mathrm{~nm}$ but no obvious fluorescence under this excitation wavelength, which may be caused by the appearance of fluorescence quenching upon IR-1061 accumulation within liposomes. ${ }^{23}$ The lowest intensity of CLPs suggested that the encapsulation of IR-1061 by liposomes was extremely poor. These results agree well with the expected effect of liposome charge on IR-1061 encapsulation. IR-1061, which is positively charged, would decrease the encapsulation effect by mutually repulsing with positively charged DOTAP, and conversely would attract the negatively charged DOPG to increase the dispersion space of liposomes in the bilayer.

Furthermore, IR-1061 encapsulated in ALPs had an absorption peak at $890 \mathrm{~nm}$. This is because IR-1061 exists in an H-aggregated state in addition to the free state in liposomes. ${ }^{24,25}$ The space within the liposomal bilayer is limited, so a high concentration of IR-1061 would induce the transition from the free state to the $\mathrm{H}$-aggregated state. For this reason, we investigated how the concentration of IR-1061 influences its state in ALPs by comparing their excitation wavelengths. Four kinds of ALPs, named IR1061ALP-N1 (N2, N3, and N4), were prepared with different contents of IR-1061 $(0.5 \%, 1 \%, 1.5 \%$, and $2 \%)$. The results demonstrated that the absorbance of IR-1061 at $1064 \mathrm{~nm}$ increased rapidly at low contents but almost no longer over $1.5 \%$ content, whereas the increase of the absorbance at $890 \mathrm{~nm}$ did not stop (Fig. 3a). By comparing the ratio of absorbance at $1064 \mathrm{~nm}$ and $890 \mathrm{~nm}$, we found that the ratio decreased continuously with the increasing concentrations of IR-1061, which indicated that the ratio of the 

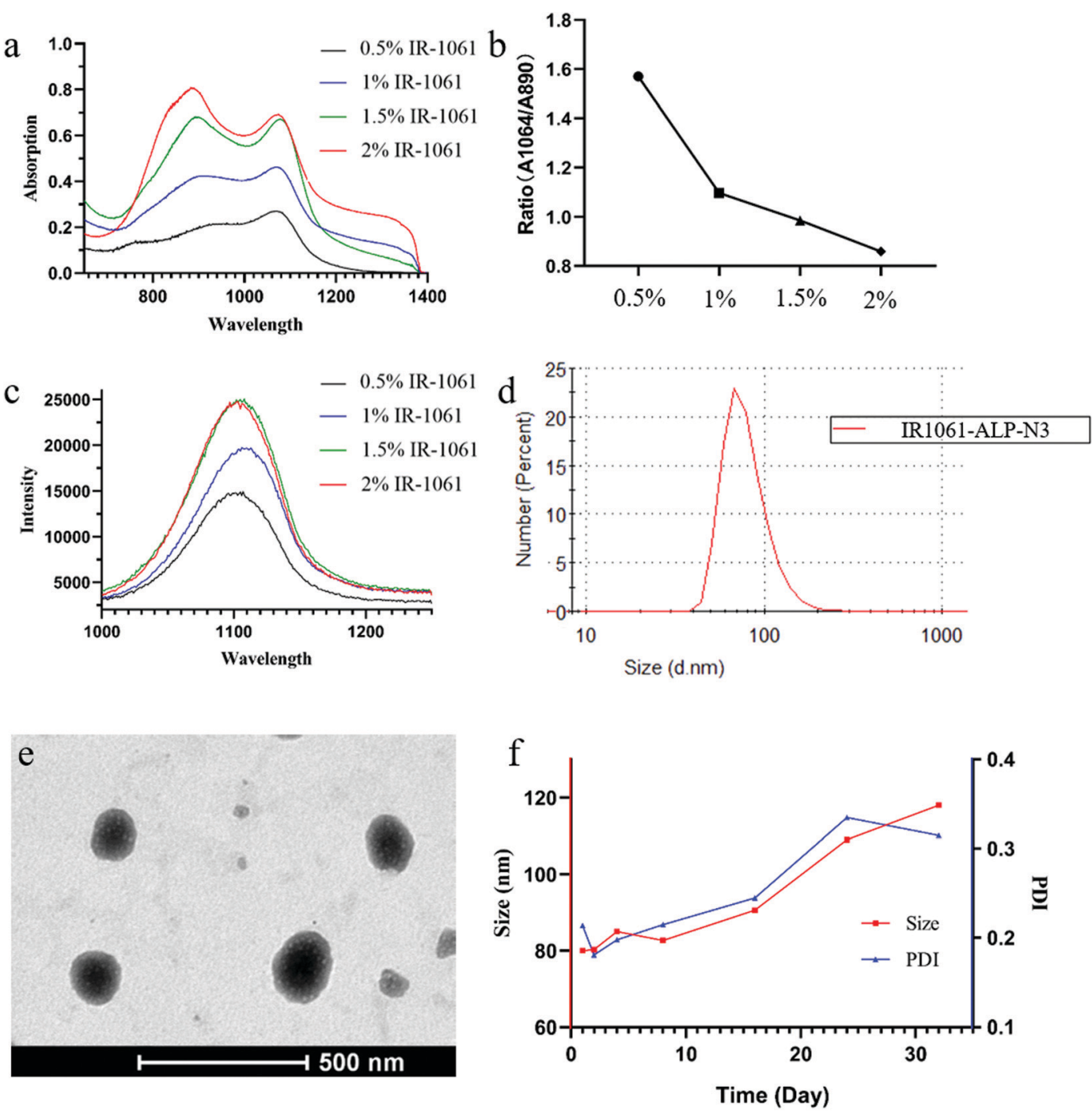

Fig. 3 Physicochemical and photophysical characterization of IR-1061 ALPs. The absorption and fluorescence spectra (a and c) of ALPs with different contents of IR-1061. (b) The ratio of absorbance of IR-1061 ALPs at $1064 \mathrm{~nm}$ and $890 \mathrm{~nm}$. (d and e) The mean hydrodynamic size and TEM of IR1061-ALP-N3. (f) The change of particle size of IR1061-ALP-N3 for 30 days.

free state to the H-aggregated state of IR-1061 also decreased (Fig. 3b). Moreover, the cessation of absorbance increase of IR1061 at $1064 \mathrm{~nm}$ showed that IR-1061 reaches its maximum in the free state at $1.5 \%$ content, implying that its fluorescence intensity might also reach its peak. Therefore, we detected the fluorescence intensity of IR-1061 at $1064 \mathrm{~nm}$ with different concentrations, the results of which were in accordance with our prediction (Fig. 3c).

In order to further study that the compression of the liposome bilayer space will promote the transition of IR-1061 from the free state to the $\mathrm{H}$-aggregated state, we prepared ALPS with different contents of IR-1061 under the condition of adjusting the concentration of phospholipids. The decrease of fluorescence intensity at $1061 \mathrm{~nm}$ indicates the occurrence of the H-aggregated state of IR-1061 in liposomes because the $\mathrm{H}$-aggregation of fluorophores will weaken the fluorescence in general (Fig. 4b). As shown in Fig. 4a, the absorption at $890 \mathrm{~nm}$ increased rapidly while the absorption at $1061 \mathrm{~nm}$ decreased significantly, demonstrating that IR-1061 in liposomes will change from the free state to the $\mathrm{H}$-aggregated state with the increase of its concentration. Temperature can also be an important factor to influence the monomer and aggregation behavior of fluorophores, so we decided to prepare IR-1061 liposomes using uncharged phospholipids with different phase transition temperatures $\left(T_{\mathrm{m}}\right)$ to study this condition. In this work, lecithin $\left(T_{\mathrm{m}}:-8{ }^{\circ} \mathrm{C}\right)$, DMPC $\left(T_{\mathrm{m}}: 23{ }^{\circ} \mathrm{C}\right)$, DPPC $\left(T_{\mathrm{m}}: 41{ }^{\circ} \mathrm{C}\right)$ and DSPC $\left(T_{\mathrm{m}}: 55{ }^{\circ} \mathrm{C}\right)$ were used to synthesise NLPs containing $2 \%$ IR-1061. The corresponding preparation temperatures were controlled to be $25{ }^{\circ} \mathrm{C}, 40{ }^{\circ} \mathrm{C}, 50{ }^{\circ} \mathrm{C}$ and $60{ }^{\circ} \mathrm{C}$. The absorption and emission spectra of the different types of NLPs showed no significant difference, demonstrating that temperature is not a major factor affecting the structural types of IR-1061 within liposomes (Fig. 4c and d).

In terms of the above experimental results, the anionic liposome with 1.5\% IR-1061 (IR1061-ALP-N3) was considered 

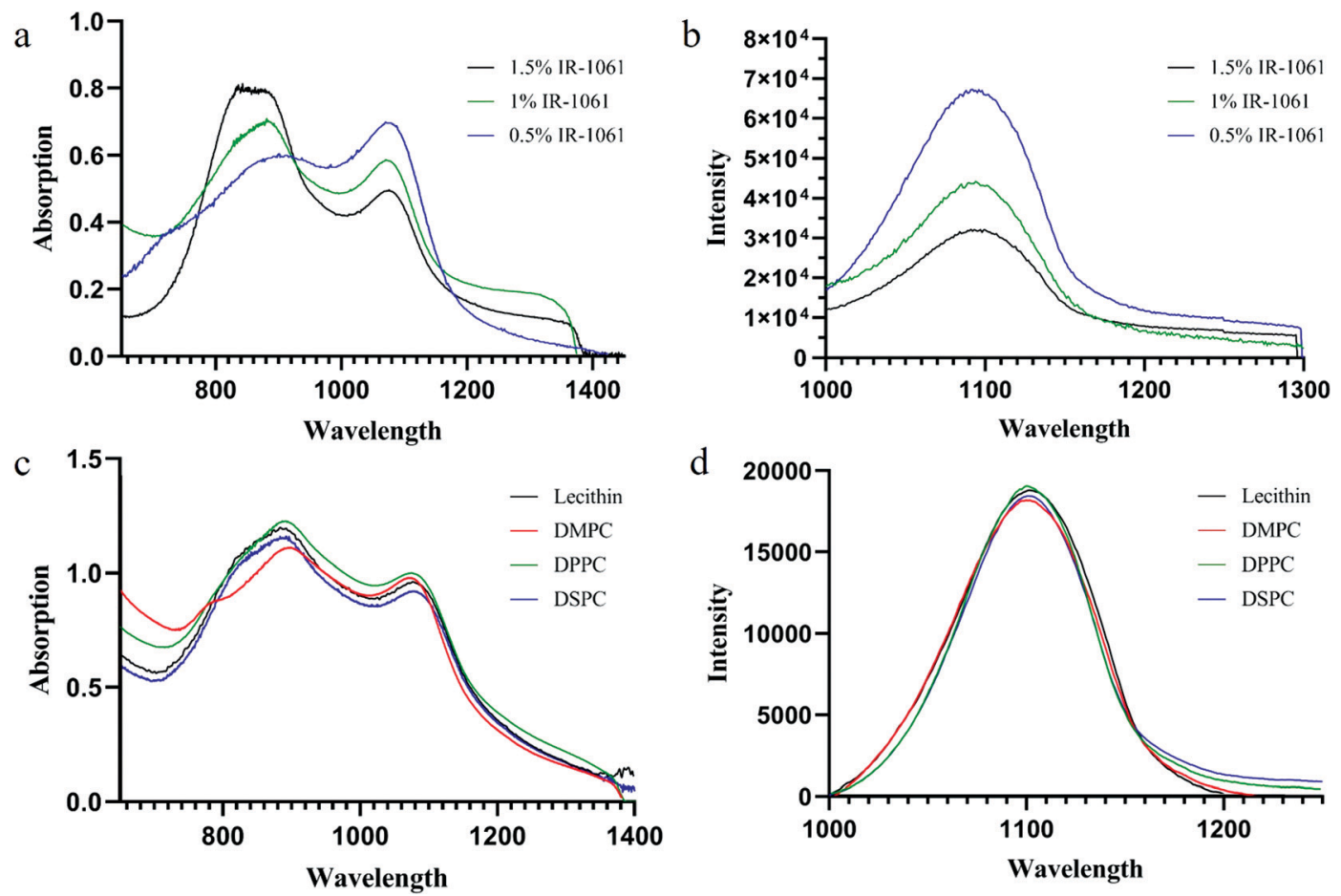

Fig. 4 Investigation on the factors affecting the fluorescence properties of IR-1061 ALPs. (a and b) The absorption and fluorescence spectra of IR-1061 ALPs with different concentrations of phospholipids. (c and d) The absorption and fluorescence spectra of IR-1061 NLPs with different phospholipids.

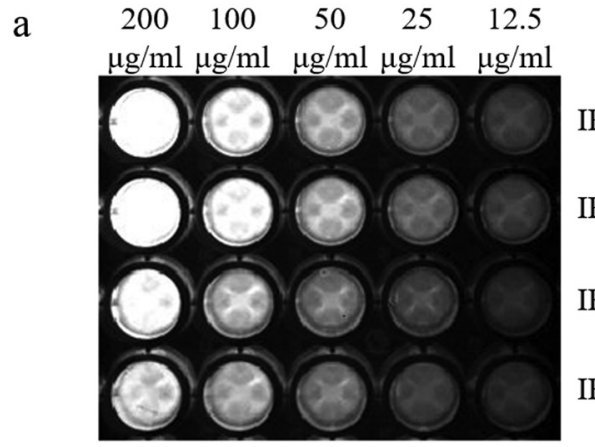

IR1061-ALP-N4

IR1061-ALP-N3

IR1061-ALP-N2

IR1061-ALP-N1

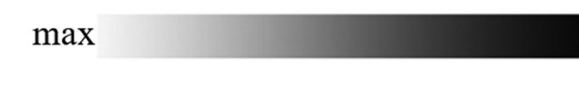

b

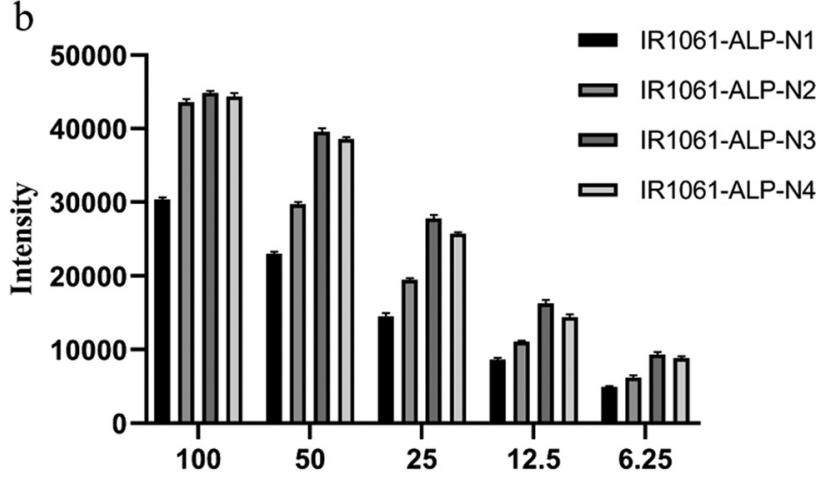

Concentration $(\mu \mathrm{g} / \mathrm{ml})$ c
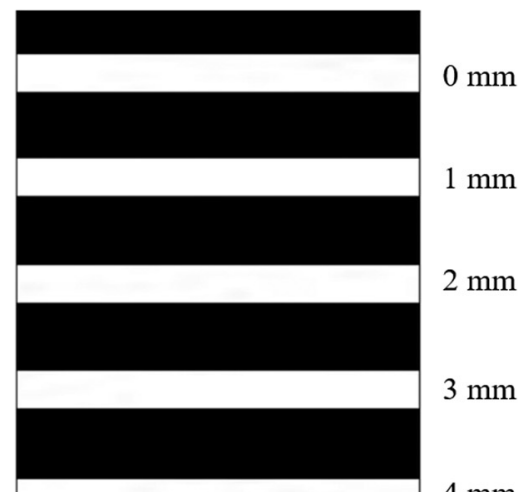

$3 \mathrm{~mm}$

$4 \mathrm{~mm}$

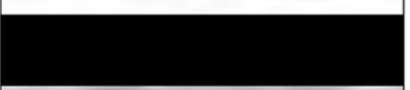

$5 \mathrm{~mm}$

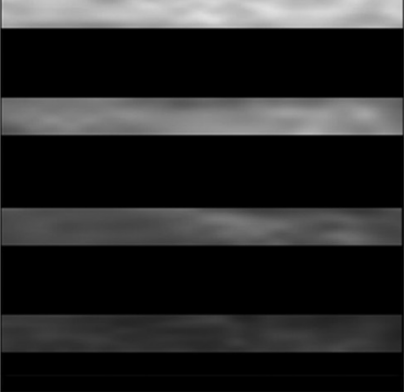

$6 \mathrm{~mm}$

$7 \mathrm{~mm}$

$8 \mathrm{~mm}$

$\max$

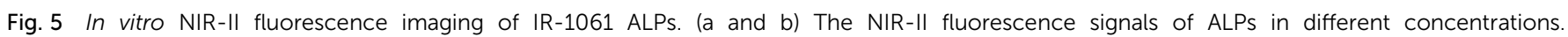
(c) The fluorescence intensity of IR1061-ALP-N3 covered with chicken tissue of different thicknesses. 


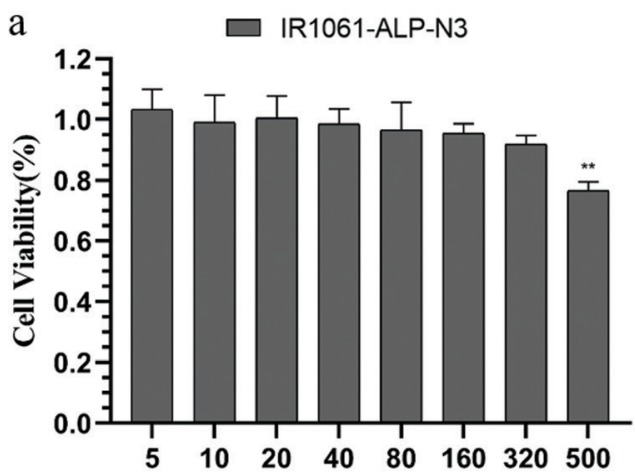

$\mathrm{b}$
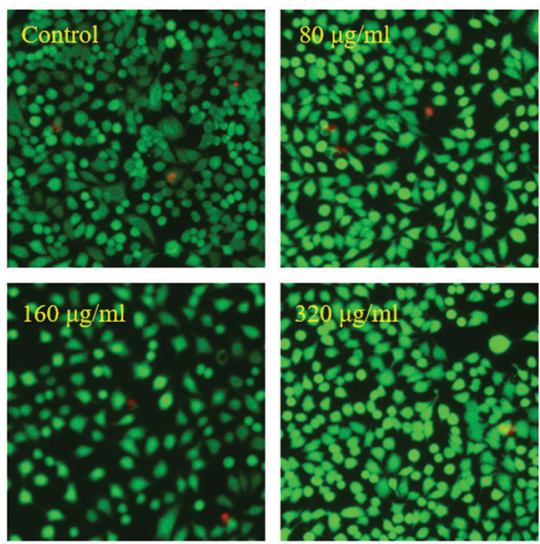

c

Concentration $(\mu \mathrm{g} / \mathrm{ml})$
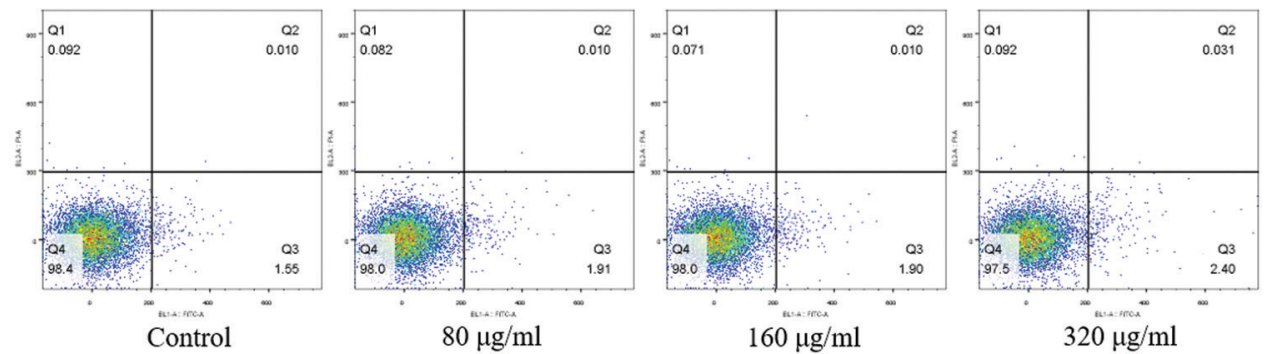

Fig. 6 Biocompatibility experiments of IR1061-ALP-N3. (a) Cell viability of A549 cells at different phospholipid concentrations of IR1061-ALP-N3. ${ }^{\star \star} P<0.01$ vs. control. (b) Fluorescent pictures of living/dead cells at different phospholipid concentrations of IR1061-ALP-N3 (green: calcein, red: propidium iodide). (c) Flow analysis of apoptotic A549 cells at different phospholipid concentrations of IR1061-ALP-N3 (abscissa: FITC, ordinate: propidium iodide).

as the optimal fluorescence liposome for subsequent studies. The mean hydrodynamic size of IR1061-ALP-N3 was $85 \mathrm{~nm}$ (PDI: 0.138) (Fig. 3d) and the TEM image is shown in Fig. 3e. The change of particle size within two weeks proved that IR1061-ALP-N3 had good stability (Fig. 3f).

\subsection{Biocompatibility experiments and in vitro NIR-II fluorescence imaging}

Next, we investigated the NIR-II fluorescence signals of ALPs in different concentrations (Fig. 5a and b). ALPs containing more than 1\% IR-1061 showed almost the same fluorescence signal at $200 \mu \mathrm{g} \mathrm{mL}$ phospholipid concentration, which represents the maximum fluorescence signal that the fluorescence system can detect. But at $100 \mu \mathrm{g} \mathrm{mL}^{-1}$ as well as lower phospholipid concentrations, IR1061-ALP-N3 showed a significantly stronger fluorescence signal than ALPs with low IR-1061 content, and slightly stronger than ALPs with 2\% IR-1061 content, indicating that IR1061-ALP-N3 has the best imaging effect for NIR-II imaging. The tissue penetration depth of IR1061-ALP-N3 was evaluated using different thicknesses of chicken tissue. As shown in Fig. 5c, IR1061-ALP-N3 had no significant decrease in tissue depth less than $5 \mathrm{~mm}$, and the maximum penetration depth could exceed $7 \mathrm{~mm}$, indicating that IR1061-ALP-N3 has a good tissue penetration depth and is suitable for in vivo fluorescence imaging.

To study the biocompatibility of IR1061-ALP-N3, the toxicity of IR1061-ALP-N3 was evaluated by cell viability and cell apoptosis detection. IR1061-ALP-N3 did not apparently decrease the activity of A549 cells and even the phospholipid concentrations up to $320 \mu \mathrm{g} \mathrm{mL}(p>0.05)$, indicating that IR1061-ALP-N3 did not interfere with the physiological activities of A549 cells (Fig. 6a). Furthermore, fluorescence signals of live/dead cells detected by fluorescence microscopy showed no significant appearance of dead cells in the experimental groups (Fig. 6b). Next, cell apoptosis was analyzed by flow cytometry. The ratio of apoptotic cells showed no difference between the experimental group and the control group when the phospholipid concentration of IR1061-ALP-N3 was lower than $320 \mu \mathrm{g} \mathrm{mL} \mathrm{m}^{-1}$, demonstrating that IR1061-ALP-N3 exhibits good biocompatibility (Fig. 6c).

\subsection{NIR-II FI properties and distribution of IR1061-ALP-N3 in vivo}

To assess the in vivo vascular FI abilities of IR1061-ALP-N3, the NIR-II fluorescence signals of the whole body and hind limb of mice were captured under $1064 \mathrm{~nm}$ excitation after a tail vein injection of IR1061-ALP-N3 at a phospholipid concentration of $2 \mathrm{mg} \mathrm{mL} \mathrm{mL}^{-1}$. As depicted in Fig. 7a, the systemic vascular structure of mice can be clearly discerned, especially the vascular imaging of the abdominal and hind limb tissues showed a significant contrast with the surrounding background tissues. The full width at half maximum (FWHM) of the abdominal and hind limb blood vessels was estimated to be $0.6 \mathrm{~mm}$ and $1.0 \mathrm{~mm}$, demonstrating that IR1061-ALP-N3 can show a high-resolution NIR-II FI in vivo (Fig. 7b and c). 
a

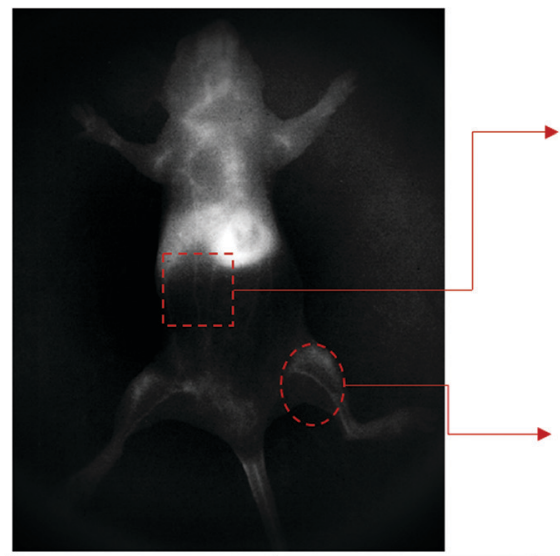

d

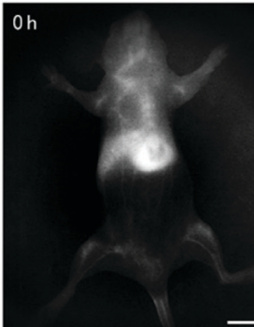

$8 \mathrm{~h}$

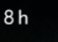

$2 \mathrm{~h}$

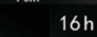

$16 \mathrm{~h}$
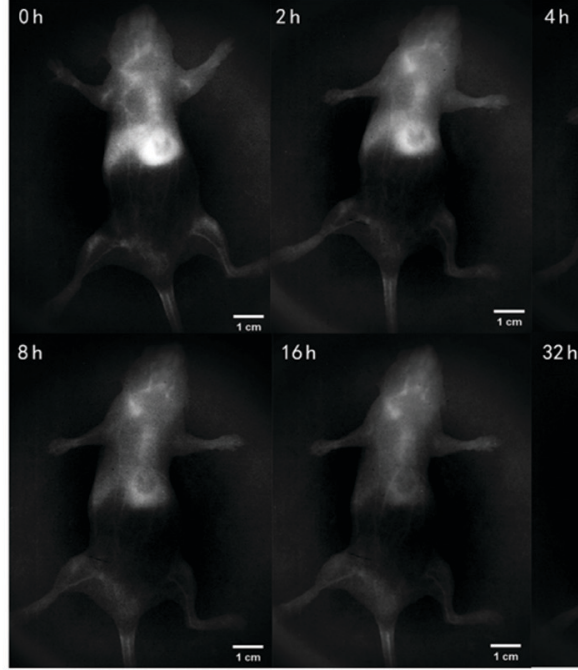
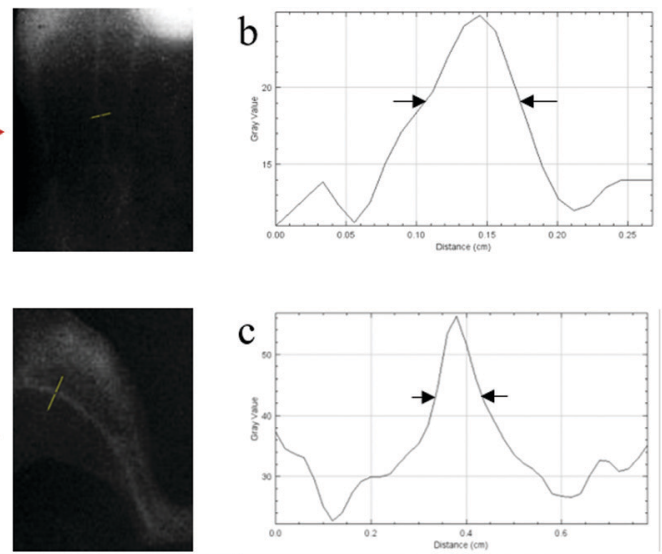

$4 \mathrm{~h}$

$32 \mathrm{~h}$
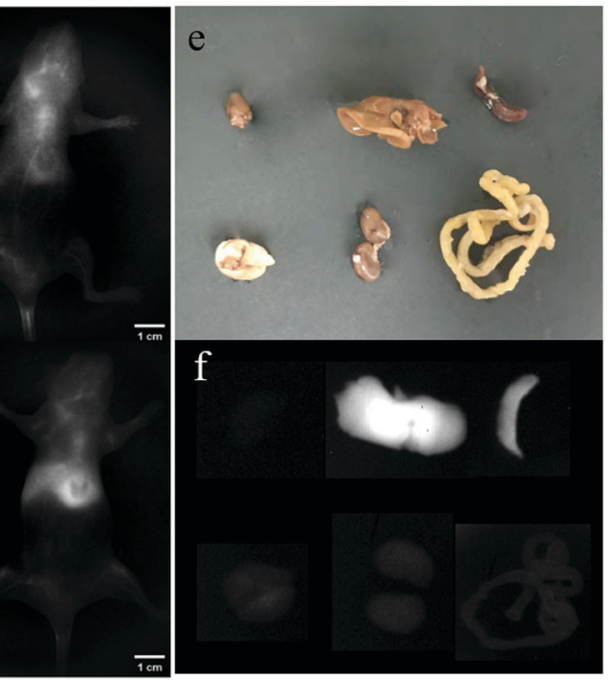

Fig. 7 NIR-II Fl properties and distribution of IR1061-ALP-N3 in vivo. (a) Systemic angiography of the Balb/c nude mice body after intravenous injection with IR1061-ALP-N3 under a $1064 \mathrm{~nm}$ laser excitation (1064 nm LP and $1064 \mathrm{~nm}$ OD filters). (b and c) Fluorescence intensities of the abdomen and hind limb vasculatures. (d) NIR-II Fls of Balb/c nude mice at various time points (0, 2, 4, 8, 16 and $32 \mathrm{~h}$ ). (e and f) Distributions of IR1061-ALP-N3 in Balb/c nude mice after $48 \mathrm{~h}$.

Drugs encapsulated by ALPs are well known to exhibit the remarkable ability of long circulation in the blood. ${ }^{26,27}$ This character can overcome the short lifetime of fluorophores in vivo. In this study, we further detected the long-time NIR-II FI of IR1061ALP-N3 (Fig. 7d). The obvious fluorescence signal could be observed after a few seconds of liposome injection, proving that IR1061-ALP-N3 had a good time resolution. The strong fluorescence signals could last up to 16 hours after liposome injection and the whole blood vessels of mice were still clearly visible, which indicates that IR1061-ALP-N3 can achieve long-term vascular imaging. After the intravenous injection for 32 hours, the resolution of whole-body angiography decreased, whereas the fluorescence intensity in the liver increased significantly, indicating that liposomes were gradually removed from the blood. After IR1061-ALP-N3 was circulated in vivo for 48 hours, the main organs of the mice (heart, liver, spleen, lungs, and kidneys) were removed and their NIR-II fluorescence signals were captured (Fig. 7e and f). A bright NIR-II fluorescence signal was mainly observed in the liver and spleen, demonstrating that IR1061-ALP-N3 was finally entrapped and cleared through the reticuloendothelial system in vivo.

\section{Discussion and conclusions}

The liposomal system is widely used to carry drugs because of its high biocompatibility and long circulation time in vivo, especially suitable for NIR-II fluorophores. However, NIR-II fluorophores are usually charged and exhibit an extremely rigid molecular structure that will strongly limit their capsulation effect. $^{28,29}$ In this study, we assumed that the charge of phospholipids can affect the encapsulation performance of liposomes and prepared three differently charged liposomes (neutral, cationic and anionic) for testing. The results showed that cationic phospholipids can hardly encapsulate IR-1061, which is due to their electrostatic repulsion. In contrast, anionic liposomes showed stronger fluorescence signals and higher encapsulation effects than neutral liposomes. Moreover, in the experimental preparation, we found that ALPs can adapt the content of IR-1061 over than 3\% under the same content of IR-1061 in NLPs or CLPs, it would appear sediment because of excessive concentration. ${ }^{30}$ These data suggested that negatively charged phospholipids can not only increase the entrapment 
efficiency of IR-1061, but also improve the distribution space of IR-1061 in the bilayer by increasing its recruitment between phospholipids under electrostatic attraction. ${ }^{31}$ We considered that there was an increase in the amount of IR-1061 on both sides of the phospholipid bilayer in ALPs, and a decrease in the amount of IR-1061 in the middle region, so aggregation and precipitation were avoided.

With the help of the characteristics between ALPs and IR-1061, we designed and prepared IR1061-ALP-N3 with optimal fluorescence performance. In vitro NIR-II FI demonstrated that IR1061-ALP-N3 possessed the highest imaging intensity over other fluorescent liposomes and a good tissue penetration depth. Cell experiments also proved that liposomes possessed good biocompatibility. These results indicate that IR1061-ALP-N3 is a suitable fluorescent probe for in vivo NIR-II FI. The next in vivo study showed that IR1061-ALP-N3 can clearly display angiography, demonstrating its excellent in vivo imaging performance. It is worth mentioning that the required probe dose (IR-1061) for angiography was significantly lower than other reports on fluorescence enhanced liposomes. $^{32}$

One of the main functions of NIR-II FI in vivo is tumor imaging. The capacity of IR1061-ALP-N3 in long-term imaging is more conducive to aggregation into tumor tissues by avoiding being captured by the macrophages and liver. However, ALPs do not easily cross vascular endothelial cells and are therefore less easily absorbed by tumors than CLPs and NLPs. ${ }^{33}$ Further modification is needed to maintain liposomes in an active state to be recognized and absorbed by tumors.

In addition, the $\mathrm{H}$-aggregated state of fluorophores will convert the light absorption from a radiative state to a nonradiative state, which will induce the photothermal conversion. $^{34,35}$ So, it can be predicted that IR1061-ALP-N3 has excellent photothermal conversion efficiency, and shows a promising application in the field of photothermal therapy of tumors and the synergistic effect of thermochemotherapy with the help of nanosystems. ${ }^{36}$

\section{Conflicts of interest}

The authors have no other relevant affiliations or financial involvement with any organization or entity with a financial interest in or financial conflict with the subject matter or materials discussed in the manuscript apart from those disclosed.

\section{Acknowledgements}

This work was supported by the National Natural Science Foundation of China (Grant number 81671745) and the Suzhou key industry technology innovation project (Grant number SYG201912).

\section{References}

1 Z. Li, Y. Xu and H. Xu, et al., A dicyanomethylene-4H-pyranbased fluorescence probe with high selectivity and sensitivity for detecting copper (II) and its bioimaging in living cells and tissue, Spectrochim. Acta, Part A, 2021, 244, 118819.

2 N. Kwon, D. Kim and K. M. K. Swamy, et al., Metal-coordinated fluorescent and luminescent probes for reactive oxygen species (ROS) and reactive nitrogen species (RNS), Coord. Chem. Rev., 2021, 427, 213581.

3 Y. Wang, X. Yan and Q. Kou, et al., An Ultrasensitive Label-Free Fluorescent Aptasensor Platform for Detection of Sulfamethazine, Int. J. Nanomed., 2021, 16, 2751-2759.

$4 \mathrm{H}$. Yu and M. Ji, Recent Advances of Organic Near-Infrared II Fluorophores in Optical Properties and Imaging Functions, Mol. Imaging Biol., 2021, 23(2), 160-172.

5 Z. Hu, C. Fang and B. Li, et al., First-in-human liver-tumour surgery guided by multispectral fluorescence imaging in the visible and near-infrared-I/II windows, Nat. Biomed. Eng., 2020, 4(3), 259-271.

6 G. Hong, A. L. Antaris and H. Dai, Near-infrared fluorophores for biomedical imaging, Nat. Biomed. Eng., 2017, 1(1), 10.

7 K. Welsher, S. P. Sherlock and H. Dai, Deep-tissue anatomical imaging of mice using carbon nanotube fluorophores in the second near-infrared window, Proc. Natl. Acad. Sci. U. S. A., 2011, 108(22), 8943-8948.

8 P. Wang, Y. Fan and L. Lu, et al., NIR-II nanoprobes in-vivo assembly to improve image-guided surgery for metastatic ovarian cancer, Nat. Commun., 2018, 9, 2898.

9 C. Hazra, S. Ullah and Y. E. Serge Correales, et al., Enhanced NIR-I emission from water-dispersible NIR-II dye-sensitized core/active shell upconverting nanoparticles, J. Mater. Chem. C, 2018, 6(17), 4777-4785.

10 Y. Cai, Z. Wei and C. Song, et al., Optical nano-agents in the second near-infrared window for biomedical applications, Chem. Soc. Rev., 2019, 48(1), 22-37.

11 R. Alford, H. M. Simpson and J. Duberman, et al., Toxicity of organic fluorophores used in molecular imaging: literature review, Mol. Imaging, 2009, 8(6), 341.

12 A. Mishra, R. K. Behera and P. K. Behera, et al., Cyanines during the 1990s: A Review, Chem. Rev., 2000, 100(6), 1973-2012.

13 A. L. Antaris, H. Chen and K. Cheng, et al., A small-molecule dye for NIR-II imaging, Nat. Mater., 2016, 15(2), 235.

14 Y. Li, J. Zhang and S. Liu, et al., Enlarging Reservoir: High Absorption Coefficient Dyes Enable Synergetic Near Infrared-II Fluorescence Imaging and Near Infrared-I Photothermal Therapy, Adv. Funct. Mater., 2021, 2102213.

15 Q. Yang, Z. Hu and S. Zhu, et al., Donor Engineering for NIR-II Molecular Fluorophores with Enhanced Fluorescent Performance, J. Am. Chem. Soc., 2018, 140(5), 1715-1724.

16 S. Wang, Y. Fan and D. Li, et al., Anti-quenching NIR-II molecular fluorophores for in vivo high-contrast imaging and pH sensing, Nat. Commun., 2019, 10, 1058.

17 Y. Su, B. Yu and S. Wang, et al., NIR-II bioimaging of small organic molecule, Biomaterials, 2021, 271, 120717.

18 F. Zhou, J. Gao and Z. Xu, et al., Overcoming immune resistance by sequential prodrug nanovesicles for promoting 
chemoimmunotherapy of cancer, Nano Today, 2021, 36, 101025.

19 M. Casalboni, F. De Matteis and P. Prosposito, et al., Fluorescence efficiency of four infrared polymethine dyes, Chem. Phys. Lett., 2003, 373(3), 372-378.

20 W. Zian, L. Yang and W. Peng, et al., Small molecular interaction-based fluorescence enhancement for second near-infrared imaging, Nanomedicine., 2020, 15(2), 115-129.

21 T. T. Duong, A. Isomäki and U. Paaver, et al., Nanoformulation and Evaluation of Oral Berberine-Loaded Liposomes, Molecules, 2021, 26(9), 2591.

22 Y. Chen, B. Sun and X. Jiang, et al., Double-acceptor conjugated polymers for NIR-II fluorescence imaging and NIR-II photothermal therapy applications, J. Mater. Chem. B, 2021, 9(4), 1002-1008.

23 K. Li, L. Wang and J. Chen, et al., Detecting methylphenethylamine vapor using fluorescence aggregate concentration quenching materials, Sens. Actuators, B, 2021, 334, 129629.

24 S. Shekhar, I. Park and J. Kim, et al., Nanoscale mapping of wavelength-selective photovoltaic responses in $\mathrm{H}$ - and $\mathrm{J}$ aggregates of azo dye-based solar cell films, J. Mater. Chem. A, 2021, 9(1), 632-641.

25 Q. Zhao, J. He and W. Yang, et al., Aggregation-induced emission characteristics and distinct fluorescent responses to external pressure stimuli based on dumbbell $D-\pi-A-\pi-D$ cyanostyrene derivatives, Tetrahedron, 2020, 76(50), 131675.

26 H. Ren, Y. He and J. Liang, et al., Role of Liposome Size, Surface Charge, and PEGylation on Rheumatoid Arthritis Targeting Therapy, ACS Appl. Mater. Interfaces, 2019, 11(22), 20304-20315.

27 Y. Yamamoto, Y. Nagasaki and Y. Kato, et al., Longcirculating poly(ethylene glycol)-poly(d,l-lactide) block copolymer micelles with modulated surface charge, J. Controlled Release, 2001, 77(1), 27-38.
28 R. Zhang, Y. Duan and B. Liu, Recent advances of AIE dots in NIR imaging and phototherapy, Nanoscale, 2019, 11(41), 19241-19250.

29 H. Yoon, H. Lee and J. Lim, et al., Liposomal Indocyanine Green for Enhanced Photothermal Therapy, ACS Appl. Mater. Interfaces, 2017, 9(7), 5683-5691.

30 Z. Chen, A. Lohr and C. R. Saha-Möller, et al., Selfassembled $\pi$-stacks of functional dyes in solution: structural and thermodynamic features, Chem. Soc. Rev., 2009, 38(2), 564-584.

31 D. Miranda, C. Wan and H. I. Kilian, et al., Indocyanine green binds to DOTAP liposomes for enhanced optical properties and tumor photoablation, Biomater. Sci., 2019, 7(8), 3158-3164.

32 W. Zhang, W. Deng and H. Zhang, et al., Bioorthogonaltargeted $1064 \mathrm{~nm}$ excitation theranostic nanoplatform for precise NIR-IIa fluorescence imaging guided efficient NIR-II photothermal therapy, Biomaterials, 2020, 243, 119934.

33 S. E. A. Gratton, P. A. Ropp and P. D. Pohlhaus, et al., The effect of particle design on cellular internalization pathways, Proc. Natl. Acad. Sci. U. S. A., 2008, 105(33), 11613-11618.

34 S. Gao, G. Wei and S. Zhang, et al., Albumin tailoring fluorescence and photothermal conversion effect of nearinfrared-II fluorophore with aggregation-induced emission characteristics, Nat. Commun., 2019, 10, 2206.

35 Q. Wang, B. Xia and J. Xu, et al., Biocompatible small organic molecule phototheranostics for NIR-II fluorescence/photoacoustic imaging and simultaneous photodynamic/photothermal combination therapy, Mater. Chem. Front., 2019, 3(4), 650-655.

36 Y. Li, J. Zhang and S. Liu, et al., Enlarging Reservoir: High Absorption Coefficient Dyes Enable Synergetic Near Infrared-II Fluorescence Imaging and Near Infrared-I Photothermal Therapy, Adv. Funct. Mater., 2021, 2102213. 